\title{
Bacteriology of the burn wound at the Bai Jerbai Wadia Hospital for children, Mumbai, India-a 13-year study, Part I-Bacteriological profile
}

\author{
Shankar Srinivasan, Arvind M. Vartak, Aakanksha Patil, Jovita Saldanha \\ Department of Burns and Plastic Surgery, B.J. Wadia Hospital for Children, Parel, Mumbai, India
}

Address for correspondence: Dr. Shankar Srinivasan, 91/22, Bhaveshwar Vijay, Wadala, Mumbai-400 031, India.

E-mail: plastic_shankar@hotmail.com

\section{ABSTRACT}

Aim: To study which organisms were prevalent in our burn unit and their antibiotic sensitivity pattern in brief. Method: Microbiological data of 1534 patients admitted to the burns unit of the Bai Jerbai Wadia Hospital for Children, Mumbai over a period of 13 years (1994-2006) was reviewed retrospectively. A total of 9333 swabs were cultured and antibiotic sensitivities to the isolated organisms determined. The age group of patients admitted to our facility ranged from one month to 15 years. Result: Klebsiella was the predominant organism in our set-up (33.91\%), closely followed by Pseudomonas (31.84\%). The antibiotic sensitivities of the isolated organisms are discussed in detail in the text. Conclusion: Every treatment facility has microorganisms unique to it and these change with time. It is therefore of paramount importance to have an in-depth knowledge of the resident organisms and their antibiotic sensitivity pattern so that infection-related morbidity and mortality are improved.

\section{KEY WORDS}

Bacteriology in burns; burn wounds; paediatric burns

\section{INTRODUCTION}

urn injury is a major problem in many parts of the world. It has been estimated that $75 \%$ of all deaths following burns are related to infection. Thermal injury destroys the skin barrier that normally prevents invasion by microorganisms. This makes the burn wound the most frequent origin of sepsis in these patients. ${ }^{[1]}$

Initially, the burnt area is considered free of microbial contamination. But gram-positive bacteria in the depth of sweat glands and hair follicles heavily colonize the wounds within $48 \mathrm{~h}$ of the injury. ${ }^{[2,3]}$

Topical antimicrobials decrease microbial overgrowth but seldom prevent further colonization with other potentially invasive bacteria and fungi. These are derived from the patient's gastrointestinal and upper respiratory tract and the hospital environment. ${ }^{[4,5]}$

Following colonization, these organisms start penetrating the viable tissue depending on their invasive capacity, local wound factors and the degree of the patient's immunosuppression..$^{[5]}$ If sub-eschar tissue is invaded, 
disseminated infection is likely to occur. ${ }^{[3]}$ Great emphasis must therefore be placed on early identification of local signs of invasive burn wound infection.

The causative infective microorganisms in any burn facility change with time. ${ }^{[6,7]}$ Individual organisms are brought into the burns ward on the wounds of new patients. These organisms then persist in the resident flora of the burn treatment facility for a variable period of time, only to be replaced by newly arriving microorganisms. Introduction of new topical agents and systemic antibiotics influence the flora of the wound. ${ }^{[6,7]}$

Thus, it is just not sufficient to be aware of the microorganisms that pose a problem for burn patients. To have an in-depth knowledge of the organisms that are predominant in that particular treatment facility during the particular period along with their sensitivity pattern is vital as many septic burn patients need to be treated with antibiotics before the results of microbiological cultures are available. This would be crucial to reduce the overall infection-related morbidity and mortality.

In the present study, we determined the nature of microbial wound colonization in 1534 patients. The major objectives were to determine:

- Which microorganisms were prevalent in our treatment facility,

- Their antibiotic sensitivity pattern.

\section{MATERIAL AND METHODS}

\section{Patients}

This is a retrospective analysis of the study of isolates from the burns unit of Bai Jerbai Wadia Hospital for children, Mumbai. The hospital caters exclusively to a paediatric population. In our study, the youngest child was a month old and the oldest, 15 years old. Between 1994 and 2006, a total of 9333 samples were processed. The sex distribution of the patients and the aetiology of burns are presented in Tables 1 and 2. It is interesting to note that in our series, male children outnumbered females by $13.2 \%$. Mortality figures are presented in Table 3. This study focuses exclusively on the microbiological profile and no attempt has been made to correlate this with clinical data. We desire to do this as a separate study.

\section{Wound treatment}

Closed dressings using silver sulphadiazine ointment were used in all patients without exception. The burn wounds were washed daily to remove necrotic tissue and the remnants of the previous day's ointment.

\section{Procedure for wound sampling}

Microbial colonization of all wounds was studied from the time of admission to discharge. On admission, the sampling procedure included swabs that were taken from clinically deep areas of the burn wound prior to any cleansing. Swabs were taken twice weekly. The bandages were removed, the remnants of the previous day's ointment were washed away and the wounds were swabbed and cultured as follows: A sterile cotton swab is moistened with sterile normal saline. This swab is rubbed onto the burn wound surface. Swabs are taken from areas which appear deep, areas with discharge, thick eschar, etc. The soabs are then sent for culture.

\section{Microbiology}

The swabs are transported to the laboratory for processing immediately. They are streaked onto a differential medium (e.g.; Mac Conkey agar] and an enriched medium (e.g.; blood agar). Isolation is carried out by the conventional T-method using sterile nichrome loop. These plates are incubated at $37{ }^{\circ} \mathrm{C}$ for $16-18 \mathrm{~h}$. The basic aim was to isolate the organisms predominant on the burn wound and determine their sensitivity to various antibiotics for clinical purposes.

Antibiotic sensitivity of isolates obtained from the burn wound was carried out by filter paper disc diffusion method (Kirby Bauer method). Sterile commercially available filter paper discs, onto which a definite amount of antibiotic has been absorbed, are used. Since the antibiotic in the disc tends to diffuse more onto the surface of the agar than into the deeper layers, the plate is surface spread with the organisms. A broth culture of the isolate is prepared using sterile peptone water comparable to 0.5 Macfarlands turbidity standard (i.e. $1 \times 10^{7}$ to $1 \times 10^{8}$ organisms $/ \mathrm{ml}$ ). Approximately $0.2 \mathrm{ml}$ of this broth culture is surface spread onto sterile Mac Conkey agar plate (for gram-negative organisms)/sterile blood agar plate (for gram-positive organisms), so as to get a matt growth.

Sterile antibiotic discs are equidistantly placed on these plates and gently pressed onto the medium with the help of sterile forceps to ensure complete contact with the agar surface. The plates are incubated at $37^{\circ} \mathrm{C}$ for 16 to $18 \mathrm{~h}$. 


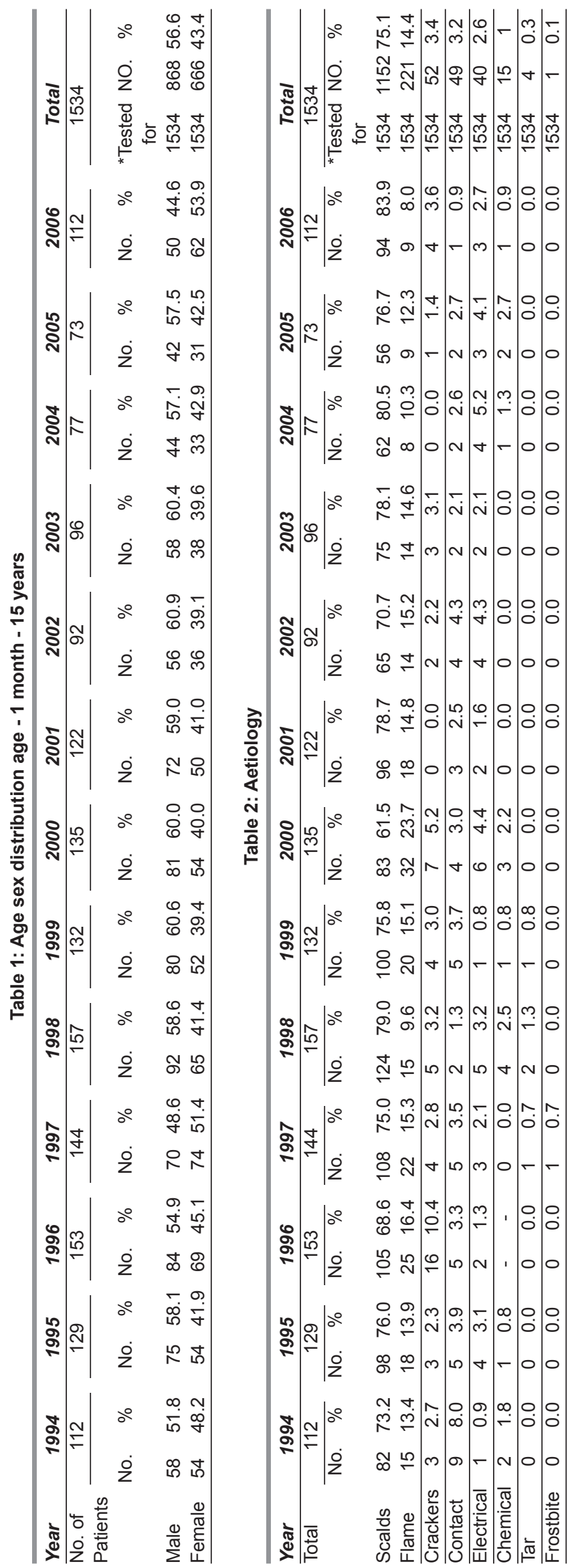

Zones of inhibition are measured in millimetres and the organisms classified as sensitive or resistant according to the zone size interpretation chart. It must be noted that our antibiotic sensitivities were not carried out on Mueller Hinton agar as advocated by some authors ${ }^{[20,21]}$. Subsequently, we have carried out a comparative study and tested antibiotic sensitivities for 10 different burn wound isolates on Mueller Hinton and Blood agar/ Mac Conkey agar and found no significant difference in the [Figure $1 \& 2$ ] results.

\section{RESULTS}

In the present study, a total of 9333 samples were processed from patients admitted to the burns unit; 1281 samples $(13.72 \%)$ showed absence of bacterial pathogens [Table 4].

The most common isolate was Klebsiella (33.91\%) followed by Pseudomonas aeruginosa (31.84\%).A detailed break-up is given in Table 5 .

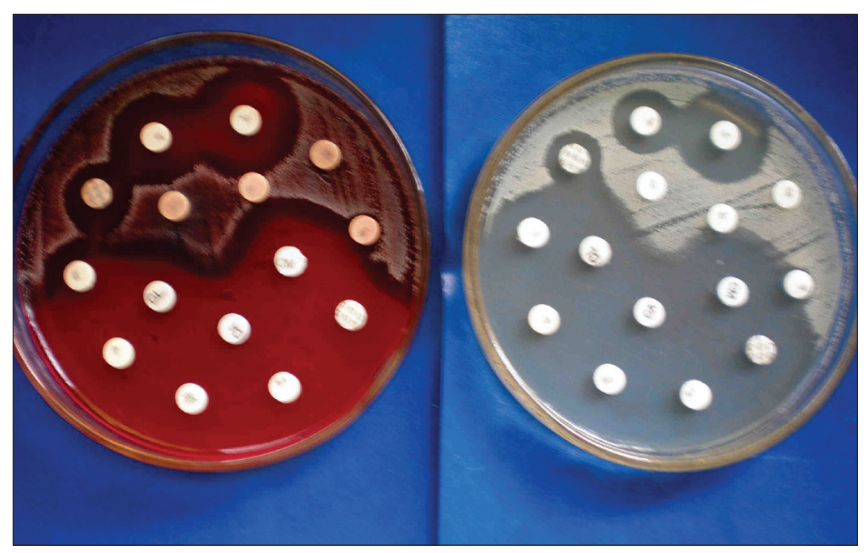

Figure 1: Comparitive study with mueller hinton agar and blood agar

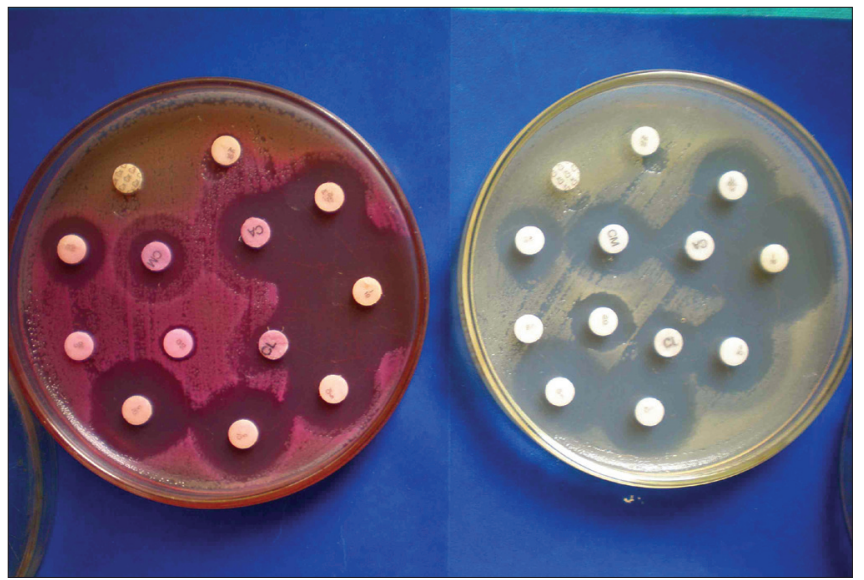

Figure 2: Comparitive study with mueller hinton and mac conkey agar 


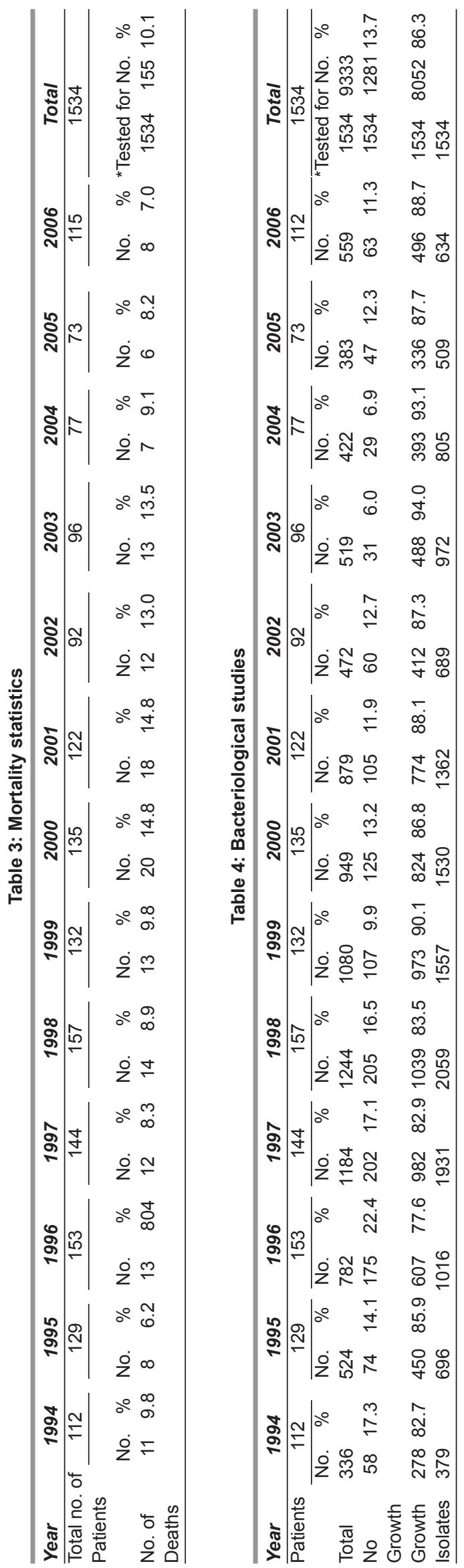

A detailed analysis on individual microorganisms and their antibiotic sensitivities, along with changing trends over this 13-year period is presented. What follows is a bird's eye view of the microorganism and its dominant sensitivity pattern.

Klebsiella was sensitive to Gatifloxacin (86.3\%)

Cefaperazone + Sulbactam (82.8\%)

Piperacillin + Tazobactam (77.4\%)

Meropenem (72.4\%)

Amikacin (66.9\%)

Azithromycin (60.4\%).

Pseudomonas was sensitive to Cefoperazone + Sulbactam (73.9\%)

Piperacillin + Tazobactam (72.2\%)

Amikacin (62.3\%)

Azithromycin (56.3\%)

Meropenem (55.8\%)

Gatifloxacin (49.9\%).

S. aureus was sensitive to Sparfloxacin (90.4\%)

Cefpirome (80.9\%)

Piperacillin+Tazobactum (78.4\%)

Netilmicin (77.2\%)

Imipenem $(64 \%)$

Erythromycin (51.1\%).

E. coli was sensitive to Ticarcillin + Clavulanic acid (67.2\%)

Meropenem (63.6\%)

Amikacin (42.7\%)

Azithromycin (27.4\%)

Gatifloxacin (61.9\%)

Cefoperazone + sulbactam (69.1\%).

Proteus was sensitive to Piperacillin+Tazobactam (97.1\%)

Meropenem (82.9\%)

Ceftrioxone and Ceftizoxime (64.6\%)

Gatifloxacin (62.9\%)

Amikacin (55.8\%)

Azithromycin (47.8\%).

\section{DISCUSSION}

Thermal injury destroys the barrier function of skin, allowing microbial colonization of wounds and even with the use of topical antimicrobials, contamination of wounds is unavoidable.

The type and amount of microorganisms on and in the injured tissue influence wound healing, ${ }^{[7]}$ the frequency 


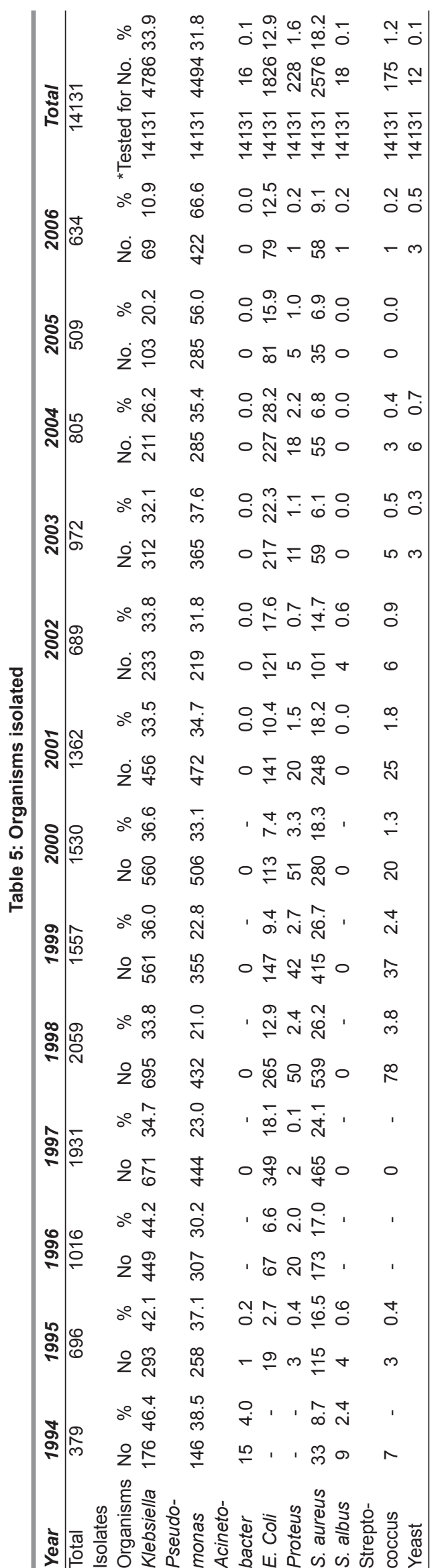

of invasive infection and the clinical characteristics of such infections as well as the risk of dissemination. Thus, knowledge of the burn ward microbial flora and the current antibiotic sensitivities at any time is important for the clinician treating burn sepsis.

It has been our observation that when patients are brought to the hospital with exposed burnt areas, the initial swabs reveal no growth. After applying a closed dressing, repeat swabs from the same patient reveal presence of microorganisms. Admittedly, burn biopsy is a better tool to determine microbiological colonization and invasion and for quantitative evaluation. It is also less fallacious. Many centres however, in our country and the world over as well, continue to rely on swab culture reports to initiate treatment as the specimens are easier to obtain and processing time comparatively lesser.

In this study, we found that the most frequent isolates were Klebsiella followed by Pseudomonas (31.84\%). Compared to several earlier reports on burn wound colonization and invasive infection, one of the most striking differences is the frequency of Klebsiella in this study, which is contrary to findings in other studies in which Klebsiella formed a small number of total isolates. ${ }^{[1,8-12]}$ It was interesting to note that two units in Nigeria ${ }^{[13,14]}$ also had Klebsiella as the most frequent pathogen isolated. The burns unit at Ain Shans University Hospital, Egypt, reported Klebsiella as the second most common organism in their study. ${ }^{[19]}$

The pattern of bacterial sensitivities is subject to frequent changes. Its assessment is important for clinical and epidemiological purposes.

For ease of discussion, the various antibiotics were grouped under their respective generic families, e.g.; penicillins, macrolides, quinolones. Antibiotics which did not fit in were placed in the "other antibiotics" group.

Data mining revealed that while Klebsiella was the dominant organism from 1994 to 2000, Pseudomonas gained the upper hand from 2001. Klebsiella was the dominant organism in 2002; subsequently, Pseudomonas became the reigning organism from 2003 to 2006 (66.6\% in 2006 while the percentage of Klebsiella is 10.9\%). It will be a revelation to us too to see how this pans out. The prevalence of Escherichia was on the rise from 2001 to 2004 and it is starting to wean off from 2005 15.9\% and $12.5 \%$ (2006) in successive years. The percentage 
incidence of Staphylococci is on the decline from 2002 to 2005.

\section{CONCLUSION}

It may be concluded that the composition of bacterial flora in burns is dependent not only on the depth and extent of the burn but also on the site of burn, the duration of burn, the age of the patient and his/her co-morbidities. ${ }^{\mid 15]}$ Burn wound monitoring requires the study of changing bacterial flora and the antibiotic sensitivity reports. Repeated swab cultures and antibiograms are advised for proper selection of antibiotics to control sepsis. ${ }^{[18]}$ The development of resistance to a particular antibiotic is dependent on the use of that antibiotic in society at large. Overuse of any antibiotic predisposes to development of resistance. Our unit gets patients from all over Mumbai, other parts of the state of Maharashtra and at times, from other states too. Due to this huge diversity, we have a particular microorganism predominant at a particular point in time, but then, it is also difficult to comment on the source of the changing trends.

\section{REFERENCES}

1. Vindenes, Jerknes. Microbial colonization of large wounds. Burns 1995;21:575-9.

2. Luterman A, Dasco CC, Curreri PW. Infection in burn patients. Am J Med 1986;81:45-52.

3. Mooney DP, Gamelli RL. Sepsis following thermal injury. Comp Ther 1989;15:22-9.

4. Monafo WW, Freedman B. Topical therapy for burns. Surg Clin North Am 1987;67:133-45.

5. Hansbrough JF. Burn wound sepsis. J Intensive Care Med 1987;2:313-27.

6. Pruitt BA Jr, McManus AT. Oppurtounistic infections in severely burnt patients. Am J Med 1984;76:146-54.
7. Manson WL, Pernot PC, Fidler V, Sauer EW. Colonisation of burns and duration of hospital stay of severely burned patients. J Hosp Infect 1992;22:55-63.

8. Revathi G, Puri J, Jain BK. Bacteriology of burns. Burns 1998;24:347-9.

9. Mehta M, Dutta P, Gupta V. Bacterial isolates from burn wound infections and their antibiograms. Indian J Plast Sugr 2007;40: 25-8.

10. Agnihotri N, Gupta V, Joshi RM. Aerobic bacterial isolates from burn wound infections and their antibiograms: A 5 year study. Burn 2004;30:241-3.

11. Ahmad M, Shahid Hussain S, Ibrahim Khan M, Malik SA. Pattern of bacterial invasion in burn patients at the Pakistan institute of medical sciences, Islamabad. Ann Burns Fire Disasters 2006;19:18-21.

12. Pezzino T, Cucchiara B, Vitale R, Benigno A, Cucchiara P, D’Arpa $\mathrm{N}$, et al. Analysis of bacteriological monitoring in patients at the Palermo Burns Centre-A 5 yr experience. Annals of the MBC-vol 2-n2-June 1989. p. 72-8.

13. Ozumba UC, Jiburum BC. bacteriology of burn wounds in Enugu, Nigeria. Burns 2000;26:178-80.

14. Kehinde AO, Ademola SA, Okesola AO, Oluwatosin OM, Bakare RA. Pattern of bacterial pathogens in burn wound infections in Ibadan, Nigeria. Ann Burns Fire Disasters 2004;18:12-5.

15. Thomsen M. The Burns unit in Copenhagen. Scand J Plast Reconst Surg 1970;4:126-39.

16. Edward-Jones V, Greenwood JE. What's new in burn microbiology? James laing Memorial Prize Essay 2000. Burns 2003;29:15-24.

17. Steer JA, Papini RP, Wilson AP, McGrouther DA, Parkhouse N. Quantitative microbiology in the management of burn patients, I: Correlation between quantitative and qualitative burn wound biopsy culture and surface alginate swab culture. Burns 1996;22:173-6.

18. Bairy I, Shivananda PG. Aerobic bacterial flora of burn wound infection. Indian J Surg 1997;59:215-8.

19. Nasser S, Mabrouk AM, Maher A. Colonisation of burn wounds in Ain Shams Univ Burns Unit. Burns 2003;29:229-33.

20. Bauer, Kirby,Sherris and Turck,1996, Am.J.Clin.Path., 45:493.

21. Performance Standards for Antimicrobial Disk Susceptibility Test, CLSI Vol. 28 No. 1, Jan. 2008.

Source of Support: Nil, Conflict of Interest: None declared. 